\title{
Amtliche Mitteilungen
}

\author{
Berichtszeitraum 20. Juli 2020 bis 23 . September 2020
}

\section{Bereich Lebensmittel}

\section{1 (a) Ausnahmegenehmigung nach $\S 68$ Absatz 1 und 2 Nr. 1 LFGB für das Behandeln und das Inverkehrbringen}

- von Schaleneiern durch direkte Einwirkung mit ultravioletten Strahlen (Bekanntmachung des BVL 111.11253.0.0054 vom 23. September 2020; GMB1 im Druck). Es handelt sich um ein Produkt der Geflügelhof Wittmer GmbH, 66839 Schmelz.

- von Schaleneiern durch direkte Einwirkung mit ultravioletten Strahlen (Bekanntmachung des BVL 111.11253.0.0055 vom 23. September 2020; GMB1 im Druck). Es handelt sich um ein Produkt der HagedornVoß Handels- und Verwaltungs GmbH, 48356 Nordwalde.

\section{2 (b) Verlängerung einer Ausnahmegenehmigung gemäß § 68 Abs. 1 und 2 Nr. 1 LFGB für das Behandeln und das Inverkehrbringen}

- von Schaleneiern durch direkte Einwirkung mit ultravioletten Strahlen (Bekanntmachung des BVL 111.11253.0.0009 vom 20. Juli 2020; GMBl im Druck). Es handelt sich um ein Produkt der Moos-Butzen GmbH, 41751 Viersen.

- von Schaleneiern durch direkte Einwirkung mit ultravioletten Strahlen (Bekanntmachung des BVL 111.11253.0.0013 vom 31. August 2020; GMB1 im Druck). Es handelt sich um ein Produkt der HennengoldEi Vertriebs GmbH \& Co. KG, 49586 Merzen.
- von Schaleneiern durch direkte Einwirkung mit ultravioletten Strahlen (Bekanntmachung des BVL 111.11253.0.0014 vom 20. Juli 2020; GMB1 im Druck). Es handelt sich um ein Produkt der Gutshof-Ei GmbH, 23795 Schackendorf.

- von Schaleneiern durch direkte Einwirkung mit ultravioletten Strahlen (Bekanntmachung des BVL 111.11253.0.0015 vom 31. August 2020; GMB1 im Druck). Es handelt sich um ein Produkt der EifrischVermarktung GmbH \& Co. KG, 49393 Lohne.

\section{3 (c) Ausnahmegenehmigung nach $\S 68$ Abs. 1} und 2 Nr. 1 LFGB für das Herstellen, Behandeln und das Inverkehrbringen

- einer mit Vitamin D angereicherten ESL Vollmilch (Bekanntmachung des BVL 111.11251.0.0082(2020) vom 21. Juli 2020; GMB1 im Druck). Es handelt sich um ein Produkt der Müller Service GmbH und der Molkerei Weihenstephan GmbH \& Co.KG, beide 85354 Freising. - von Nahrungsergänzungsmitteln mit Zusatz von Kollagen (Bekanntmachung des BVL 111.11256.0.0150 vom 22. Juli 2020; GMBl im Druck). Es handelt sich um ein Produkt der PHARMA ALDENHOVEN GmbH \& Co. KG, 52449 Aldenhoven, und der ALDI SÜD Dienstleistungs-GmbH \& Co. oHG, 45476 Mühlheim an der Ruhr.

Publisher's Note Springer Nature remains neutral with regard to jurisdictional claims in published maps and institutional affiliations. 